\title{
Application of Optimal Pulse Design in Non-ideal Ultra-wideband Transmission
}

\author{
Jens Timmermann ${ }^{1}$, Alireza Ajami Rashidi ${ }^{2}$, Philipp Walk ${ }^{3}$, Elena Pancera ${ }^{4}$, and Thomas Zwick ${ }^{5}$ \\ ${ }^{1,2,4,5}$ Institut für Höchstfrequenztechnik und Elektronik, Universität Karlsruhe (TH), Germany \\ ${ }^{3}$ Institut für Telekommunikationssysteme, Technische Universität Berlin, Germany
}

\begin{abstract}
The performance of an impulse radio system depends on the pulse shape, modulation, coding, the frontend components, the channel and the receiver structure. In this contribution, a Time Hopping Pulse Position Modulation (THPPM) system is investigated that operates in an indoor scenario and consists of non-ideal frontend components and a correlation receiver. The question arises how the pulse shape influences the system performance, namely the bit error rate. Classical pulse shapes do not fully exploit the power spectral density regulation and lead to reduced transmit power and performance. Therefore, optimal pulse shapes are of great interest. This contribution first summarizes how optimal pulse shapes can be obtained and demonstrates in a second step how they improve the system performance if the system is assumed to be non-ideal which is neglected in most contributions.
\end{abstract}

Keywords - UWB, TH-PPM, impulse radio, optimal pulse design, non-ideal frontend, bit error rate

\section{INTRODUCTION}

In the FCC regulation, a bandwidth of $7.5 \mathrm{GHz}$ is available for ultra-wideband transmission, and according to Shannon's capacity equation, capacity and hence data rate linearly increases with bandwidth. For this reason, ultra-wideband techniques are seen as a candidate to realize high data rates in the order of up to $500 \mathrm{Mbit} / \mathrm{s}$. In principle, two different techniques exist to exploit the available bandwidth: OFDM based UWB which divides the bandwidth into a set of channels or impulse radio based UWB where a pulse with a bandwidth of several $\mathrm{GHz}$ is directly given to the transmit antenna. In this contribution, impulse radio is considered. It is however extremely difficult to realize an impulse radio system that operates ideally in the whole frequency range. Frontend hardware such as antennas, filters, amplifiers are non-ideal versus frequency in some sense which can degrade the system performance. Furthermore, classical pulse shapes do not fully exploit the regulation and lead to further losses in the SNR and therefore to reduced performance. Therefore, one way to improve the performance is the use of optimal pulses. This can be achieved by a pulse shaper based on a FIR (finite impulse response) filter. Section II summarizes three methods to find optimal FIR coefficients and analyzes the respective power efficiency. In Section III, the modeling of the non-ideal system is presented. Then, both classical and optimal pulse shapes are used in the non-ideal system, and the respective performance is investigated in terms of bit error rate (BER) versus signal-to-noise-ratio (SNR) and distance. Section IV gives a conclusion and an outlook.

\section{OPTIMAL PULSE DESIGN}

Classical pulse shapes like Gaussian pulses do not fully exploit the FCC regulation and lead to reduced transmit power: Fig. 1 presents the $6^{\text {th }}$ derivative of a Gaussian pulse both in time domain and frequency domain together with the indoor and outdoor regulation. The power efficiency of a pulse is defined as the power of the pulse inside the relevant range from 3.1 to $10.6 \mathrm{GHz}$ divided by the maximal allowed power of $0.56 \mathrm{~mW}$. Higher side lobe levels outside the relevant range hence do not influence the efficiency. Power efficiency is also called normalized effective signal power (NESP). In Fig. 1, the power efficiency is only $44.5 \%$.

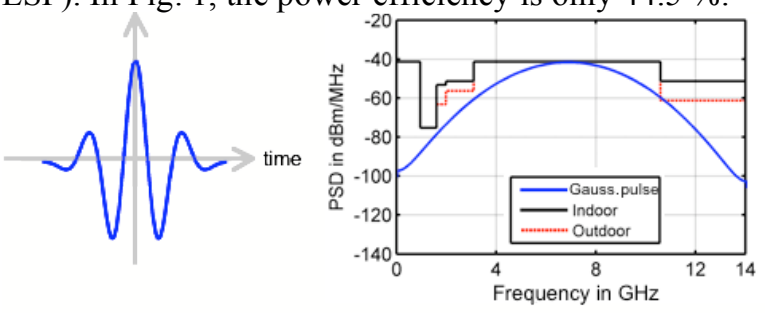

Fig. 1 Gaussian pulse ( $6^{\text {th }}$ derivative) and its power spectral density

To improve the power efficiency, pulse shaping methods using a FIR filter can be applied [1]. Optimal coefficients of the FIR filter must be found so that the impulse response of the FIR filter represents the optimal pulse shape. In the following, three methods to find optimal coefficients are summarized: window method, frequency sampling method and direct maximization of NESP [2], [3].

\section{A. WINDOW METHOD AND \\ FREQUENCY SAMPLING METHOD}

Fig. 2 shows the principle of the window method and the frequency sampling method.

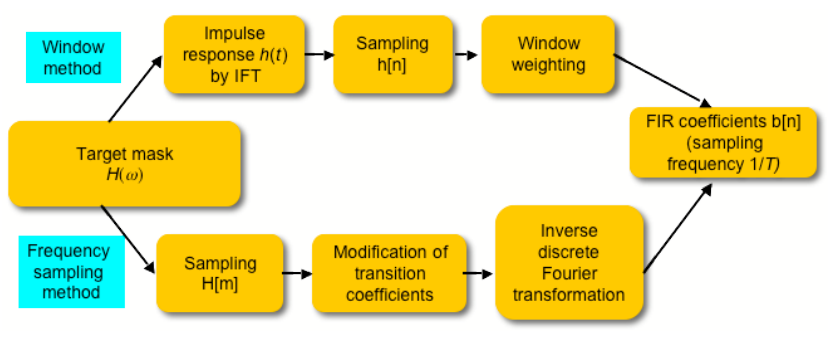

Fig. 2 Window and frequency sampling method 
For the window method, an inverse Fourier Transform (IFT) of the target mask is performed. Then, sampling in time domain gives discrete values $\mathrm{h}[\mathrm{n}]$. To reduce Gibbs phenomenon, a suited window function is used. The result are the optimal FIR coefficients.

For the frequency sampling method, the target mask is sampled in frequency domain. At edges of the target mask, transition coefficients are defined. Afterwards, a discrete inverse Fourier transform gives the optimal FIR coefficients.

Both methods are applied for a filter order of $L=66$ so that 66 FIR coefficients are determined. The target mask is the FCC mask. Fig. 3 and 4 show the resulting optimal pulse in time domain and the power spectral density (PSD) for the window method. Here a Chebyshev window is used.

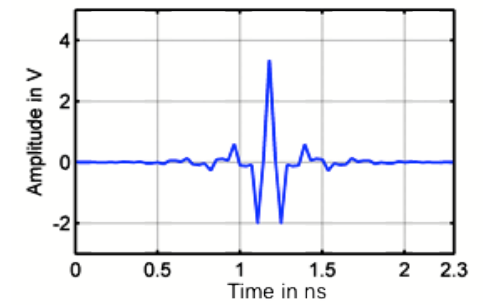

Fig. 3 Optimal pulse using window method

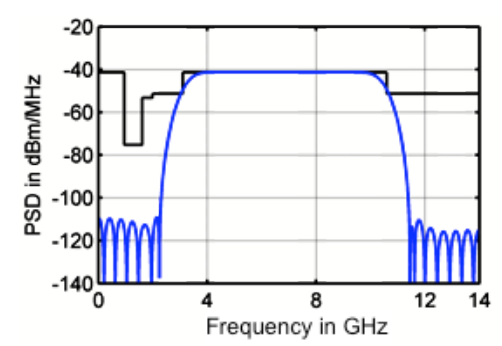

Fig. 4 PSD of optimal pulse using window method

It can be seen that the pulse fits well the desired mask. The full pulse width at half maximum is about 80 ps. The power efficiency is $88.2 \%$ which is much better than the power efficiency of the $6^{\text {th }}$ derivative of the Gaussian pulse.

For comparison, Fig. 5 and 6 show also the results for the frequency sampling method.

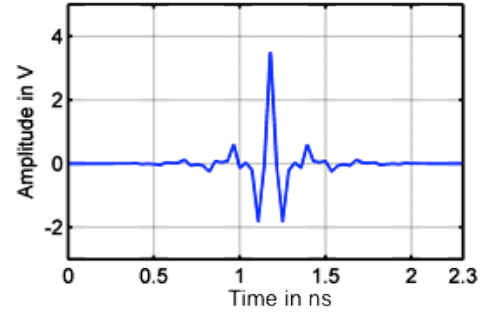

Fig. 5 Optimal pulse using frequency sampling method

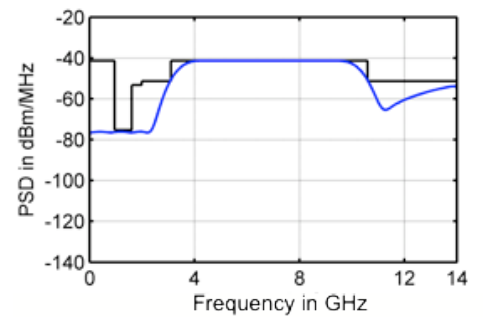

Fig. 6 PSD of optimal pulse using frequency sampling method

For sampling in frequency domain, a frequency step of $1 \mathrm{MHz}$ is used. Again, a high power efficiency (88.6\%) is achieved.

\section{B. DIRECT MAXIMIZATION OF NESP}

An alternative to window and frequency sampling method is the direct maximization of NESP. If $S_{\mathrm{p}}(f)$ denotes the PSD of the optimal pulse to be searched and $S_{\mathrm{FCC}}(f)$ the PSD of the FCC mask, the following condition must be fulfilled in the relevant frequency range:

$$
\left|S_{\mathrm{p}}(f)\right| \leq\left|S_{\mathrm{FCC}}(f)\right|
$$

$S_{\mathrm{p}}(f)$ can be expressed as the Fourier transform of the autocorrelation $R_{\mathrm{xx}}$ of the pulse according to Eq. (2):

$$
S_{\mathrm{p}}(f)=\int_{-\infty}^{\infty} R_{\mathrm{xx}}(\tau) \cdot e^{-j 2 \pi \cdot f \cdot \tau} d \tau
$$

In discrete domain, the integral reduces to a sum. With the sampling time $T_{0}$, filter order $L$, the relationship $\tau=k T_{0}$ and the symmetry of $R_{\mathrm{xx}},(2)$ can be simplified as

$$
S_{\mathrm{p}}(f)=1+2 \cdot \sum_{k=1}^{L-1} R_{\mathrm{xx}}(k) \cdot e^{-j 2 \pi \cdot f \cdot k T_{0}}
$$

Defining

$$
\begin{aligned}
V(f, L)= & {\left[1,2 \cos \left(2 \pi \cdot f \cdot T_{0}\right), 2 \cos \left(2 \pi \cdot f \cdot 2 T_{0}\right),\right.} \\
& \left.\ldots, 2 \cos \left(2 \pi \cdot f \cdot(L-1) T_{0}\right)\right]^{T}
\end{aligned}
$$

the absolute value of $S_{\mathrm{p}}(f)$ can be expressed as

$$
\left|S_{\mathrm{p}}(f)\right|=V(f, L) \cdot R_{\mathrm{xx}}(L)
$$

Equ. (5) can be solved for $R_{\mathrm{xx}}(L)$. Taking the special case $S_{\mathrm{p}}(f)=S_{\mathrm{FCC}}(f)$, the autocorrelation of the optimal pulse $R_{\text {xx_opt }}(L)$ is determined. A factorization of $R_{\text {xx_opt }}(L)$ delivers the optimal pulse shape. Fig. 7 and 8 show the optimal pulse shape and its PSD (again for the filter order of 66) using the method of direct maximization of NESP. 


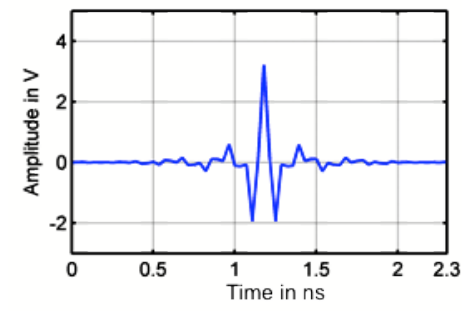

Fig. 7 Optimal pulse using Direct Maximization of NESP

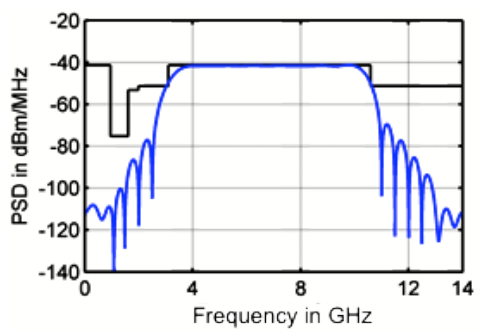

Fig. 8 PSD of optimal pulse using Direct Maximization of NESP

The power efficiency of the pulse is $84.8 \%$.

\section{NON-IDEAL SYSTEM MODELING AND SYSTEM SIMULATION}

Both classical and optimal pulse shapes are used in a nonideal impulse radio system that is modeled in the Advanced Design System (ADS). Fig. 9 shows the system model. A PPM modulated bit stream with Time Hopping coding is given to a non-ideal transmit antenna (monocone) that is modeled by its frequency dependent 3-dimensional measured pattern. The channel is modeled by Ray Tracing and represents an indoor lab scenario where transmitter (Tx) and receiver $(\mathrm{Rx})$ heights are the same. Fig. 10 shows the system model and the measured antenna pattern. AWGN interference is also added, and the signal is received by a non-ideal receive antenna (again monocone) and amplified by a commercially available non-ideal LNA before being demodulated by a correlation receiver. Details about the system model can be found in [4] and [5].

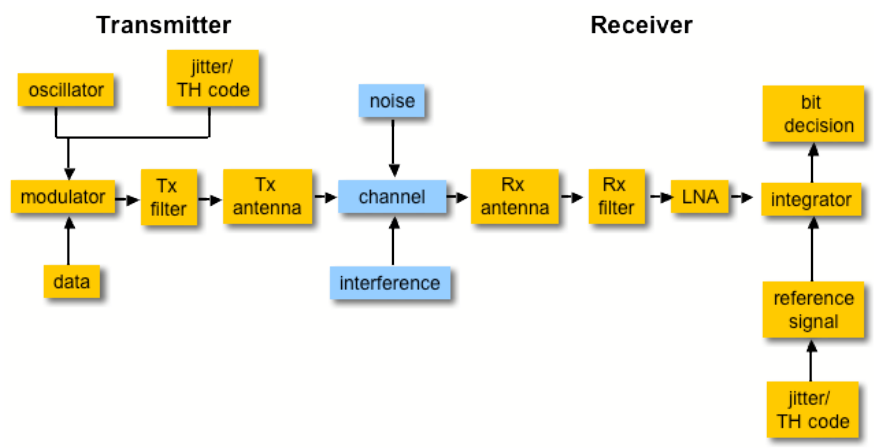

Fig. 9 System model of non-ideal UWB transmission

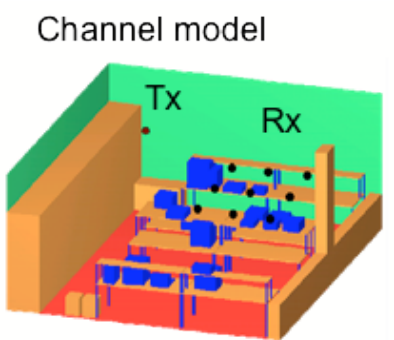

Measured antenna pattern

Fig. 10 Channel model (9 receiver positions in a Ray Tracing based lab scenario) and antenna model (measured elevation pattern)

To compare the performance of classical Gaussian pulse $\left(6^{\text {th }}\right.$ derivative $)$ and the three optimal pulse shapes, a constant data rate of $17 \mathrm{Mbit} / \mathrm{s}$ is chosen. Furthermore, for a constant distance of $2.98 \mathrm{~m}$ and a given pulse shape, 5 different interference power levels from $-80 . .-40 \mathrm{dBm}$ are chosen to get 5 different receiver SNR values. 10000 bits are transmitted for simulation. Fig. 11 visualizes the respective bit error rate versus signal-to-noise-ratio. All curves represent the same behavior, but the SNR range differs between the curves. For example, the inefficient Gaussian pulse leads to the worst receiver SNR values since the transmit power was worst. The worst SNR of the Gaussian pulse is $-37.5 \mathrm{~dB}$ in Fig. 11 and the worst SNR of the optimal pulses is $-34.5 \mathrm{~dB}$; so the difference is $3 \mathrm{~dB}$. This difference corresponds to the difference $\Delta P_{d B m}$ in $\mathrm{dBm}$ of the respective transmit power which is

$$
\begin{aligned}
\Delta P_{d B m}= & 10 \log \left(\frac{88.6 \% \cdot 0.56 \mathrm{~mW}}{1 \mathrm{~mW}}\right)- \\
& 10 \log \left(\frac{44.5 \% \cdot 0.56 \mathrm{~mW}}{1 \mathrm{~mW}}\right)=3 \mathrm{dBm}
\end{aligned}
$$

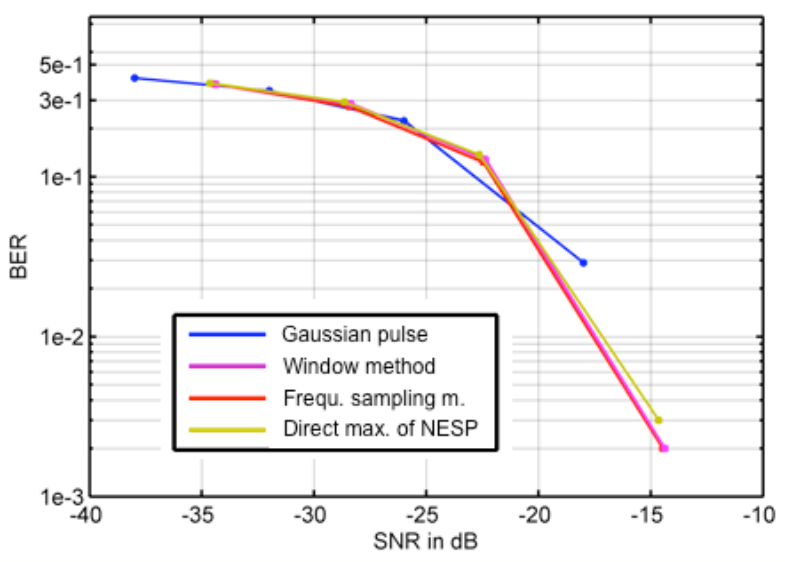

Fig. 11 Bit error rate versus SNR for classical Gaussian pulse $\left(6^{\text {th }}\right.$ derivative $)$ and optimal pulse shapes

To get the behavior of the bit error rate versus the distance, 9 different receiver positions are taken while the interference power is fixed. Fig. 12 shows the result both for the classical Gaussian pulse and the optimal pulses. The improvement of the BER by optimal pulse shapes is best for small distances, since small distances lead to good SNR values, and for good 
SNR values, an improvement of the SNR leads to a much better BER (see Fig. 11).

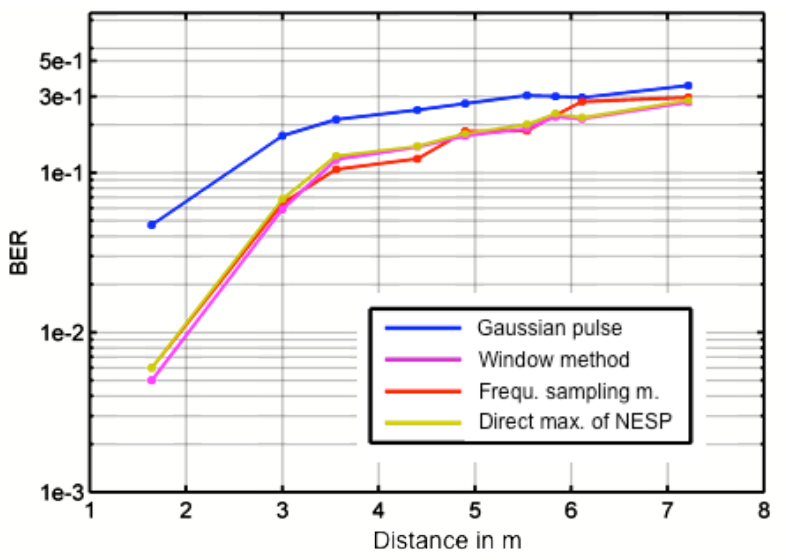

Fig. 12 Bit error rate versus distance for classical Gaussian pulse $\left(6^{\text {th }}\right.$ derivative $)$ and optimal pulse shapes

\section{CONCLUSIONS}

This paper has treated three techniques to obtain optimal pulse shapes. The power efficiency was compared to the classical Gaussian pulse ( $6^{\text {th }}$ derivative), and the impact of the pulse shape on the system performance was investigated using a realistic non-ideal ultra-wideband system model that is based on measurement data. It could be shown that optimal pulse shapes have significant advantages for small distances. To compensate the frequency dependence of the non-ideal frontend, namely the transmit antenna, the pulse shapes can be further predistorted, for example by weighting the pulses in frequency domain with the inverse antenna gain function or by defining a modified target mask that takes into account the inverse frequency dependence of the following components. For both methods, first successful results have been achieved. They will be further investigated and presented in following contributions.

\section{REFERENCES}

[1] D. Zeng, "Pulse Shaping Filter Design and Interference Analysis in UWB Communication Systems", dissertation, Faculty of The Bradley Department of Electrical and Computer Engineering, Virgina Polytechnic Institute and State University, July 2005.

[2] X. Wu, Z. Tian, T. Davison, G. Giannakis, "Optimal Waveform Design for UWB Radios", IEEE Transactions on Signal Processing, vol. 54, no. 6, pp. 2009-2021, June 2006.

[3] Z. Tian, T. Davison, X. Luo, X. Wu, G. Giannakis, "Ultra Wideband Wireless Communication", chapter 5 (Ultra Wideband Pulse Shaper Design), Wiley, 2006.

[4] J. Timmermann, E. Pancera, P. Walk, W. Wiesbeck, T. Zwick, "Bit error rate of a non-ideal impulse radio system", Proceedings of European Electromagnetics Symposium (EUROEM), Lausanne, Switzerland, Jul. 2008.

[5] J. Timmermann, E. Pancera, G. Adamiuk, W. Wiesbeck, and T. Zwick, "Estimated Performance of UWB Impulse Radio Transmission Including Dirty RF Effects", IEEE International Conference on UltraWideband, Hannover, Sept. 2008. 